\title{
ON O-MINIMAL HOMOTOPY GROUPS
}

\author{
ELÍAS BARO ${ }^{1}$ AND MARGARITA OTERO ${ }^{2}$
}

\begin{abstract}
We work over an o-minimal expansion of a real closed field. The o-minimal homotopy groups of a definable set are defined naturally using definable continuous maps. We prove that any two semialgebraic maps which are definably homotopic are also semialgebraically homotopic. This result together with the study of semialgebraic homotopy done by H. Delfs and M. Knebusch allows us to develop an o-minimal homotopy theory. In particular, we obtain o-minimal versions of the Hurewicz theorems and the Whitehead theorem.
\end{abstract}

\section{Introduction}

Many aspects of o-minimal topology have been studied in the last years. However, there has been a lack of development of o-minimal homotopy, only the first homotopy group was considered. The first aim of this paper is to fill this gap. On the other hand, a much more complete development of semialgebraic homotopy theory was carried out by H. Delfs and M. Knebusch in $[8]$.

Let $\mathcal{R}$ be an o-minimal expansion of a real closed field. We shall prove that any two semialgebraic maps which are definably homotopic are also semialgebraically homotopic (see Theorem 3.1 for the full statement). To do this, we will follow the scheme of the proofs of the results in [8], however the core of their proofs cannot be adapted to our context since they make use of both the polynomial description of semialgebraic sets and the Lebesgue number, which are not available in the o-minimal setting. Instead, we use the results on normal triangulations in o-minimal structures obtained in [1].

By applying both Theorem 3.1 and semialgebraic homotopy, we are able to develop o-minimal homotopy. In section 4, the (higher) homotopy groups are defined and their usual propeties are proved. We also prove the following transfer result (Corollary 4.4): if $X$ is a semialgebraic set defined without parameters and $X(\mathbb{R})$ is its realization over the reals, then for each $n \geq 1$, the $n$th o-minimal homotopy group of $X$ is isomorphic to the (classical) $n$th homotopy group of $X(\mathbb{R})$, this was done for the case $n=1$ in [4]. Further applications of Theorem 3.1 can be founded in [3], where the homotopy of definable groups is studied. The work of Woerheide in [13] was the beginning of the study of o-minimal homology. Here, we link the o-minimal homology groups with the corresponding homotopy ones, via the o-minimal version of

\footnotetext{
${ }^{1}$ Partially supported by GEOR MTM2008-00272.

${ }^{2}$ Partially supported by GEOR MTM2008-00272 and Grupos UCM 910444.

Date: January 27, 2009

Mathematics Subject Classification 2000: 03C64, 14P10, 55Q99.
} 
the Hurewicz theorems which are proved in section 5. Also the o-minimal version of Whitehead theorem is proved in the latter section. However, quite often to be able to apply the Whitehead theorem we need to go out of the definable category. As a further application of Theorem 3.1 we shall extend in [2] the results of this paper to the locally definable category in which the Whitehead theorem fully applies.

M. Shiota has announced some unpublished related results also linking the semialgebraic and the o-minimal topology (personal communication). After a preliminary version of this paper was written, the preprint [12] by A. Piękosz has appeared with some related results.

The results of this paper are part of the first author's Ph.D. dissertation.

Acknowledgements. We thank the referee for the careful reading of the paper.

\section{Preliminaries}

For the rest of the paper we fix an o-minimal expansion $\mathcal{R}$ of a real closed field $R$. We always take 'definable' to mean 'definable in $\mathcal{R}$ with parameters', except otherwise stated. We take the order topology on $R$ and the product topology on $R^{n}$ for $n>1$. All maps are assumed to be continuous.

We use the notation from [6] concerning simplicial complexes. Given a definable set $S$ and some definable subsets $S_{1}, \ldots, S_{l}$ of $S$ we say that $(K, \phi)$ is a triangulation in $R^{p}$ of $S$ partitioning $S_{1}, \ldots, S_{l}$ if $K$ is a simplicial complex formed by a finite number of (open) simplices in $R^{p}$ and $\phi:|K| \rightarrow S$ is a definable homeomorphism such that each $S_{i}$ is the union of the images by $\phi$ of some simplices of $K$ (note the difference with the notation in [6] for triangulations). Recall that given a subset $A$ of $|K|$, the star of $A$ in $K$, denoted by $\operatorname{St}_{K}(A)$, is the union of all the simplices $\sigma \in K$ such that $\bar{\sigma} \cap A \neq \emptyset$.

If $X$ is a definable set and $A_{1}, \ldots, A_{k}$ are definable subsets of $X$ then $\left(X, A_{1}, \ldots, A_{k}\right)$ is called a system (or pair, if $k=1$ ) of definable sets. A definable map $f:\left(X, A_{1}, \ldots, A_{k}\right) \rightarrow\left(Y, B_{1}, \ldots, B_{k}\right)$ between systems of definable sets is a definable map $f: X \rightarrow Y$ such that $f\left(A_{i}\right) \subset B_{i}$ for each $i=1, \ldots, k$.

Given a definable set $X$ we denote its frontier by $\partial X:=\bar{X} \backslash X$. We also use the standard notation $I:=[0,1]=\{x \in R: 0 \leq x \leq 1\}$.

If a set $X$ is definable with parameters in some structure $\mathcal{M}$ we denote by $X(M)$ the realization of $X$ in $\mathcal{M}$.

Lemma 2.1 (o-minimal homotopy extension lemma). Let $X, Z$ and $A$ be definable sets with $A \subset X$ closed in $X$. Let $f: X \rightarrow Z$ be a definable map and $H: A \times I \rightarrow Z$ a definable homotopy such that $H(x, 0)=f(x)$, $x \in A$. Then there exists a definable homotopy $G: X \times I \rightarrow Z$ such that $G(x, 0)=f(x), x \in X$, and $\left.G\right|_{A \times I}=H$. 
Proof. Let $(K, \phi)$ be a triangulation of $X$ partitioning $A$ and let $K_{A}=\{\sigma \in$ $K: \phi(\sigma) \subset A\}$. Observe that $\left|K_{A}\right|$ is closed in $|K|$. By Theorem 5.1 in [7], there exists a semialgebraic retract $r:|K| \times I \rightarrow\left(\left|K_{A}\right| \times I\right) \cup(|K| \times\{0\})$. This retract naturally induces a definable retract $r^{\prime}: X \times I \rightarrow(A \times I) \cup(X \times\{0\})$. Let $H^{\prime}:(A \times I) \cup(X \times\{0\}) \rightarrow Z$ be the following definable map

$$
H^{\prime}(x, t)=\left\{\begin{array}{l}
H(x, t) \text { for all } \quad(x, t) \in A \times I, \\
f(x) \text { for all }(x, 0) \in X \times\{0\} .
\end{array}\right.
$$

Then $G=H^{\prime} \circ r^{\prime}$ is the required homotopy.

Given two simplicial complexes $K$ and $L$ we say that $g:|K| \rightarrow|L|$ is a simplical map if it is the restriction to $|K|$ of a map $\widetilde{g}:|\bar{K}| \rightarrow|\bar{L}|$ which sends each simplex of $\bar{K}$ to a simplex of $\bar{L}$ by a linear map taking vertices to vertices and each simplex in $K$ to a simplex in $L$. Given a definable map $f:|K| \rightarrow|L|$ between the realizations of two simplicial complexes $K$ and $L$, with $K$ closed, we say that a simplicial map $g:|K| \rightarrow|L|$ is a simplicial approximation to $f$ if $f\left(S t_{K}(w)\right) \subset S t_{L}(g(w))$ for each $w \in \operatorname{Vert}(K)$. Note that if a simplicial complex $L$ is the first barycentric subdivision of another one then every simplex of $\bar{L}$ whose vertices lies in $L$ is a simplex of $L$. As in the classical case of closed simplices we obtain the following.

Remark 2.2. Let $K$ and $L$ be simplicial complexes, with $K$ closed, and let $f:|K| \rightarrow|L|$ be a definable map.

(i) If $g:|K| \rightarrow|L|$ is a simplicial approximation to $f$ then $f$ and $g$ are canonically definably homotopic via the map $(x, s) \mapsto(1-s) f(x)+s g(x)$ for all $(x, s) \in|K| \times I$.

(ii) If $f$ satisfies the star condition, i.e, there is $\varphi: \operatorname{Vert}(K) \rightarrow \operatorname{Vert}(L)$ such that $f\left(S t_{K}(v)\right) \subset S t_{L}(\varphi(v))$ for every vertex $v \in \operatorname{Vert}(K)$ and moreover $L$ is the first barycentric subdivision of some simplicial complex, then $f$ has a simplicial approximation, namely, the simplicial map induced by $\varphi$.

We shall use the following notion introduced in [1] (see Definition 1.3 therein). Given a closed simplicial complex $K$ in $R^{m}$ and $S_{1}, \ldots, S_{l}$ definable subsets of $|K|$ we say that $\left(K^{\prime}, \phi^{\prime}\right)$ is a normal triangulation of $K$ partitioning $S_{1}, \ldots, S_{l}$, if it satisfies the following conditions:

(i) $\left(K^{\prime}, \phi^{\prime}\right)$ is a triangulation of $|K|, \phi^{\prime}:\left|K^{\prime}\right| \rightarrow|K|$, partitioning $S_{1}, \ldots, S_{l}$ and all $\sigma \in K$,

(ii) $K^{\prime}$ is a subdivision of $K$ (in particular $\left|K^{\prime}\right|=|K|$ ), and

(iii) for every $\tau \in K^{\prime}$ and $\sigma \in K$, if $\tau \subset \sigma$ then $\phi^{\prime}(\tau) \subset \sigma$.

Fact 2.3. (i)(Normal Triangulation Theorem) Let $K$ be a closed simplicial complex and let $S_{1}, \ldots, S_{l}$ be definable subsets of $|K|$. Then there exists a normal triangulation $\left(K^{\prime}, \phi^{\prime}\right)$ of $K$ partitioning $S_{1}, \ldots, S_{l}$.

(ii) Let $\left(K^{\prime}, \phi^{\prime}\right)$ be a normal triangulation of a closed simplicial complex $K$ 
partitioning the definable subsets $S_{1}, \ldots, S_{l}$ of $|K|$. Then $i d_{|K|}$ and $\phi^{\prime}$ are canonically definably homotopic via the map $(x, s) \mapsto(1-s) x+s \phi^{\prime}(x)$, for all $(x, s) \in\left|K^{\prime}\right| \times I$.

For the proof of (i) and (ii) see Theorem 1.4 and the proof of Theorem 1.1 in Section of [1], respectively.

Extending a given triangulation is a technical tool used in the construction of triangulations (see Lemma II.4.3 in [8]). We next prove that the extension process can be done preserving normality. We will make use of this tool in the proof of Theorem 3.1.

Lemma 2.4. Let $K$ be a closed simplicial complex and $K_{Z}$ a closed simplicial subcomplex of $K$. Let $\left(K_{0}, \phi_{0}\right)$ be a normal triangulation of $K_{Z}$. Then there exists a normal triangulation $\left(K^{\prime}, \phi^{\prime}\right)$ of $K$ such that $K_{0} \subset K^{\prime}$ and $\left.\phi^{\prime}\right|_{\left|K_{0}\right|}=\phi_{0}$.

Proof. Note that $\left|K_{0}\right|=\left|K_{Z}\right|$, since $K_{0}$ is a subdivision of $K_{Z}$. For every $m \geq 0$ we denote by $S K^{m}$ the closed complex which is the union of $K_{Z}$ and all the simplices of $K$ of dimension $\leq m$. We will show that there exists a normal triangulation $\left(K^{m}, \phi^{m}\right)$ of $S K^{m}$ such that $K_{0} \subset K^{m}$ and $\left.\phi^{m}\right|_{\left|K_{0}\right|}=\phi_{0}$. Hence for $m=\operatorname{dim}(K)$ we will obtain the required normal triangulation.

For $m=0$ let $K^{0}$ be the union of $K_{0}$ and all vertices of $K$. Let $\phi^{0}$ be equal to $\phi_{0}$ on $\left|K_{0}\right|$ and the identity on the vertices of $K$ that does not lie in $\left|K_{0}\right|$. Clearly $\left(K^{0}, \phi^{0}\right)$ is a normal triangulation of $S K^{0}, K_{0} \subset K^{0}$ and $\left.\phi^{0}\right|_{\left|K_{0}\right|}=\phi_{0}$.

Suppose we have constructed $\left(K^{m}, \phi^{m}\right)$. Let $\Sigma_{m+1}$ be the collection of simplices in $K \backslash K_{0}$ of dimension $m+1$. Hence, for every $\sigma \in \Sigma_{m+1}, \partial \sigma$ is contained in $S K^{m}$. On the other hand, $K^{m}$ is a subdivision of $S K^{m}$ and so, for each $\sigma \in \Sigma_{m+1}$, there exists a finite collection of indices $J_{\sigma}$ and simplices $\tau_{j}^{\sigma}$ of $K^{m}, j \in J_{\sigma}$, such that $\partial \sigma=\dot{\bigcup}_{j \in J_{\sigma}} \tau_{j}^{\sigma}$. For each $j \in J_{\sigma}$ denote by $\left[\tau_{j}^{\sigma}, \hat{\sigma}\right]$ the cone over $\tau_{j}^{\sigma}$ with vertex the barycentre $\hat{\sigma}$ of $\sigma$, that is, $\left[\tau_{j}^{\sigma}, \hat{\sigma}\right]=\left\{(1-t) u+t \hat{\sigma}: u \in \tau_{j}^{\sigma}, t \in[0,1]\right\}$. For each $\sigma \in \Sigma_{m+1}$ and $j \in J_{\sigma}$ we define

$$
\begin{aligned}
h_{j}^{\sigma}: & {\left[\tau_{j}^{\sigma}, \hat{\sigma}\right] } \\
(1-t) u+t \hat{\sigma} & \rightarrow(1-t) \phi^{m}(u)+t \hat{\sigma} .
\end{aligned}
$$

Note that $h_{j}^{\sigma}$ is well-defined because given $u \in \tau_{j}^{\sigma}$ there exists a proper face $\sigma_{0} \in K$ of $\sigma$ such that $\tau_{j}^{\sigma} \subset \sigma_{0}$ and therefore, since $\sigma_{0} \in S K^{m}$ and $\left(K^{m}, \phi^{m}\right)$ is a normal triangulation, we have that $\phi^{m}(u) \in \phi^{m}\left(\tau_{j}^{\sigma}\right) \subset \sigma_{0} \subset$ $\partial \sigma$. Hence $h_{j}^{\sigma}((1-t) u+t \hat{\sigma}) \in \bar{\sigma}$ for all $t \in[0,1]$ and $u \in \tau_{j}^{\sigma}$. Note that the map $h_{j}^{\sigma}$ is injective and it is indeed continuous. Let $K^{m+1}$ be the collection of simplices in $K^{m}$ together with the collection of simplices $\left(\tau_{j}^{\sigma}, \hat{\sigma}\right)=\left\{(1-t) u+t \hat{\sigma}: u \in \tau_{j}^{\sigma}, t \in(0,1)\right\}$ and all their faces for $\sigma \in \Sigma_{m+1}$ and $\tau_{j}^{\sigma}$ as described above. Finally, let $\phi^{m+1}$ be the extension of $\phi^{m}$ to $K^{m+1}$ such that $\left.\phi^{m+1}\right|_{\left[\tau_{j}^{\sigma}, \hat{\sigma}\right]}=h_{j}^{\sigma}$. We show that $\phi^{m+1}$ is well-defined. It is 
enough to prove that for a fixed $\sigma \in \Sigma_{m+1}$, the sets $h_{j}^{\sigma}\left(\left(\tau_{j}^{\sigma}, \hat{\sigma}\right)\right), j \in J_{\sigma}$, are pairwise disjoint. Indeed, $h_{j}^{\sigma}\left(\left(\tau_{j}^{\sigma}, \hat{\sigma}\right)\right)=\left(\phi^{m}\left(\tau_{j}^{\sigma}\right), \hat{\sigma}\right)$, where $\left(\phi^{m}\left(\tau_{j}^{\sigma}\right), \hat{\sigma}\right)=$ $\left\{(1-t) x+t \hat{\sigma}: x \in \phi^{m}\left(\tau_{j}^{\sigma}\right), t \in(0,1)\right\}$ and since the sets $\phi^{m}\left(\tau_{j}^{\sigma}\right)$ are pairwise disjoint, the sets $\left(\phi^{m}\left(\tau_{j}^{\sigma}\right), \hat{\sigma}\right)$ are also pairwise disjoint. Note that $\phi^{m+1}$ is continuous.

We now show that $\left(K^{m+1}, \phi^{m+1}\right)$ is a normal triangulation of $S K^{m+1}$. To prove that $\left(K^{m+1}, \phi^{m+1}\right)$ partitions the simplices of $S K^{m+1}$ it is enough to consider each $\sigma \in \Sigma_{m+1}$ (since $K^{m} \subset K^{m+1},\left.\phi^{m+1}\right|_{\left|K^{m}\right|}=\phi^{m}$, and $\left(K^{m}, \phi^{m}\right)$ is normal). Now, for each of these $\sigma \in \Sigma_{m+1}$, the image of $h_{j}^{\sigma}$ is contained in $\bar{\sigma}$ and, since $\partial \sigma=\bigcup_{j \in J_{\sigma}} \phi^{m}\left(\tau_{j}^{\sigma}\right)$, we have $\sigma=\bigcup_{j \in J_{\sigma}}\left(\phi^{m}\left(\tau_{j}^{\sigma}\right), \hat{\sigma}\right) \cup$ $\{\hat{\sigma}\}$. Clearly $K^{m+1}$ is a subdivision of $S K^{m+1}$ because for the relevant simplices of $S K^{m+1}$, i.e, those $\sigma \in \Sigma_{m+1}$, the cones $\left(\tau_{j}^{\sigma}, \hat{\sigma}\right)$ and their faces form a triangulation of $\bar{\sigma}$. Also property (iii) of normality holds, since we have always worked inside each simplex $\sigma \in \Sigma_{m+1}$.

\section{The o-minimal homotopy sets}

Let $(X, A)$ and $(Y, B)$ be two pairs of definable sets. Let $C$ be a relatively closed definable subset of $X$ and let $h: C \rightarrow Y$ be a definable map such that $h(A \cap C) \subset B$. We say that two definable maps $f, g:(X, A) \rightarrow(Y, B)$ with $\left.f\right|_{C}=\left.g\right|_{C}=h$, are definably homotopic relative to $h$, denoted by $f \sim_{h} g$, if there exists a definable map $H:(X \times I, A \times I) \rightarrow(Y, B)$ such that $H(x, 0)=f(x), H(x, 1)=g(x)$ for all $x \in X$ and $H(x, t)=h(x)$ for all $x \in C$ and $t \in I$. The o-minimal homotopy set of $(X, A)$ and $(Y, B)$ relative to $h$ is the set

$[(X, A),(Y, B)]_{h}^{\mathcal{R}}=\left\{f: f:(X, A) \rightarrow(Y, B)\right.$ definable in $\left.\mathcal{R},\left.f\right|_{C}=h\right\} / \sim_{h}$.

If $C=\emptyset$ we omit all references to $h$. We shall denote by $\mathcal{R}_{0}$ the field structure of the real closed field $R$ of our o-minimal structure $\mathcal{R}$. Note that if we take $\mathcal{R}$ to be $\mathcal{R}_{0}$ above, then we obtain the definition of a semialgebraic homotopy set (see Section 2 of Chapter 3 in [8]).

Our main result is the following theorem.

Theorem 3.1. Let $(X, A)$ and $(Y, B)$ be two pairs of semialgebraic sets with $X$ closed and bounded. Let $C$ be a closed semialgebraic subset of $X$ and $h: C \rightarrow Y$ a semialgebraic map such that $h(A \cap C) \subset B$. Then, if $A$ is closed in $X$, the map

$$
\begin{aligned}
\rho:[(X, A),(Y, B)]_{h}^{\mathcal{R}_{0}} & \rightarrow[(X, A),(Y, B)]_{h}^{\mathcal{R}} \\
{[f] } & \mapsto[f]
\end{aligned}
$$

is a bijection.

We are specially interested in the case $C=\emptyset$. However, in order to reduce Theorem 3.1 to the following proposition, we will need to consider the general case. 
Proposition 3.2. Let $K, K_{C}$ and $L$ be simplicial complexes with $K$ closed and $K_{C}$ a closed subcomplex of $K$. Let $h:\left|K_{C}\right| \rightarrow|L|$ be a semialgebraic map. Then the map

$$
\rho:[|K|,|L|]_{h}^{\mathcal{R}_{0}} \rightarrow[|K|,|L|]_{h}^{\mathcal{R}}
$$

is surjective.

Granted the proposition, Theorem 3.1 is proved as follows. We first make two reductions: (i) it suffices to prove that $\rho$ is onto and (ii) it suffices to consider the case $A=B=\emptyset$. These two reductions are done in the proof of Theorem 4.2 of Chapter III in [8], pp. 250. Though the statement of Theorem 4.2 in [8] differs from our Theorem 3.1 (because a real closed field $S$ extension of $R_{0}$ is considered there instead of our o-minimal $\mathcal{R}$ ), the proof of the mentioned reductions apply to our context except that at some point they use the semialgebraic homotopy extension lemma and we have to use our o-minimal homotopy extension lemma (see Lemma 2.1). Finally, applying the semialgebraic Triangulation Theorem we can reduce to realizations of simplicial complexes.

Proof of Proposition 3.2. First note that we can assume that $L$ is the first barycentric subdivision of some simplicial complex. Let $[f] \in[|K|,|L|]_{h}^{\mathcal{R}}$. We will find a semialgebraic map definably homotopic to $f$ relative to $h$. By the proof of Theorem 4.2 of Chapter III in [8], p.254, we can assume that

(a) there exist subcomplexes $K_{D}$ of $K$ and $K_{E}$ of $K_{D}$ such that

$$
\left|K_{C}\right| \subset \operatorname{int}_{|K|}\left(\left|K_{E}\right|\right) \subset\left|K_{E}\right| \subset \operatorname{int}_{|K|}\left(\left|K_{D}\right|\right), \text { and }
$$

(b) the map $f$ satisfies $\left.f\right|_{\left|K_{D}\right|}=\widetilde{h}$, where $\widetilde{h}:\left|K_{D}\right| \rightarrow|L|$ is a semialgebraic map such that $\left.\widetilde{h}\right|_{\left|K_{C}\right|}=h$ and for each simplex $\sigma \in K_{D}$ there is a simplex of $L$ containing $\widetilde{h}(\sigma)$.

As above, even though the statement of Theorem 4.2 in [8] differs from ours, since extensions of real closed fields are considered there, the proof of the fact that we can assume (a) and (b) apply to our context using the o-minimal homotopy extension lemma (see Lemma 2.1) instead of the semialgebraic one. These assumptions allow us to protect $\left|K_{C}\right|$ with two successives "barriers", $\left|K_{D}\right|$ and $\left|K_{E}\right|$. We shall use these barriers in two different places in the following proof to transform the map $f$ without modifying it on $\left|K_{C}\right|$.

We divide the proof in two steps. In Step 1 we will make use of the Normal triangulation theorem (see Fact 2.3) to show that there exists a definable map $g$ satisfying the star condition such that $f \sim_{h} g$. In Step 2 we will use a simplicial approximation to $g$ (whose existence is ensured by the star condition) to find a semialgebraic map definably homotopic to $f$ relative to $h$. 
Step 1: Let $K_{Z}$ be the closed subcomplex of $K$ whose polyhedron is $\left|K_{Z}\right|=|K| \backslash \operatorname{int}_{|K|}\left(\left|K_{D}\right|\right)$. By the Normal Triangulation Theorem (see Fact $2.3)$ there exists a normal triangulation $\left(K_{0}, \phi_{0}\right)$ of $K_{Z} \cup K_{E}$ partitioning $f^{-1}(\sigma) \cap\left|K_{Z}\right|, \sigma \in L$. Moreover, since $\left|K_{E}\right| \cap\left|K_{Z}\right|=\emptyset$ and $\left(K_{E}\right.$, id) is a normal triangulation of $K_{E}$, we can assume that $\left.\phi_{0}\right|_{\left|K_{E}\right|}=$ id. Next we extend $\left(K_{0}, \phi_{0}\right)$ to a triangulation of the whole of $|K|$. By Lemma 2.4 there exists a normal triangulation $\left(K^{\prime}, \phi^{\prime}\right)$ of $K$ such that $K_{0} \subset K^{\prime}$ and $\left.\phi^{\prime}\right|_{\left|K_{0}\right|}=\phi_{0}$. In particular, $\left.\phi^{\prime}\right|_{\left|K_{E}\right|}=$ id. Note that $\left(K^{\prime}, \phi^{\prime}\right)$ partitions the sets $f^{-1}(\sigma), \sigma \in L$. Indeed, it suffices to show that for each $\sigma^{\prime} \in K^{\prime}, \phi^{\prime}\left(\sigma^{\prime}\right)$ is contained in the preimage by $f$ of some simplex of $L$. If $\sigma^{\prime} \subset\left|K_{Z}\right|$ this is clear since $\phi^{\prime}$ extends $\phi_{0}$, which in turn partitions the subsets $f^{-1}(\sigma) \cap\left|K_{Z}\right|$ for $\sigma \in L$. On the other hand, if $\sigma^{\prime} \subset|K| \backslash\left|K_{Z}\right| \subset\left|K_{D}\right|$ then $\phi^{\prime}\left(\sigma^{\prime}\right)$ is contained in some simplex of $K_{D}$ because $\left(K^{\prime}, \phi^{\prime}\right)$ partitions the simplices of $K$ and, by (b), each simplex of $K_{D}$ is contained in the preimage by $f$ of some simplex of $L$.

By Fact $2.3, \phi^{\prime}$ and $\operatorname{id}_{\left|K^{\prime}\right|}$ are definably homotopic via the canonical homotopy $H_{1}:\left|K^{\prime}\right| \times I \rightarrow|K|:(x, s) \mapsto(1-s) x+s \phi^{\prime}(x)$. Let $H_{2}:=f \circ H_{1}$ and $g:=f \circ \phi^{\prime}$. Since $H_{1}(x, s)=x$ for all $(x, s) \in\left|K_{E}\right| \times I, H_{2}$ is a homotopy from $f$ to $g$ relative to $\left.\widetilde{h}\right|_{\left|K_{E}\right|}$. Note also that since $\left(K^{\prime}, \phi^{\prime}\right)$ partitions $f^{-1}(\tau)$ for $\tau \in L$ we have that for every $\sigma \in K^{\prime}$ there exists $\tau \in L$ such that $g(\sigma) \subset \tau$. This implies that for every $v \in V \operatorname{ert}\left(K^{\prime}\right)$ there exist $w \in \operatorname{Vert}(L)$ with $w \in L$ such that $g\left(S t_{K^{\prime}}(v)\right) \subset S t_{L}(w)$. Indeed, take $v \in \operatorname{Vert}\left(K^{\prime}\right)$ and $\tau \in L$ such that $g(v) \in \tau$. Since $L$ is the first barycentric subdivision of some simplicial complex, there exists a vertex $w$ of $\tau$ with $w \in L$. Since $g^{-1}\left(S t_{L}(w)\right)$ is the realization of a subcomplex of $K^{\prime}$, it is open in $\left|K^{\prime}\right|$ and contains the vertex $v$, we deduce that $S t_{K^{\prime}}(v) \subset g^{-1}\left(S t_{L}(w)\right)$.

Step 2: Consider the map $\mu_{\text {vert }}: \operatorname{Vert}\left(K^{\prime}\right) \rightarrow \operatorname{Vert}(L): v \mapsto \mu_{\text {vert }}(v)$, where (as in Step 1) $\mu_{\text {vert }}(v)$ is such that $\mu_{\text {vert }}(v) \in L$ and $g\left(\operatorname{St}_{K^{\prime}}(v)\right) \subset$ $\mathrm{St}_{L}\left(\mu_{\mathrm{vert}}(v)\right)$. By Remark 2.2(ii) the map $\mu_{\mathrm{vert}}$ induces a simplicial approximation $\mu$ to $g$. However neither $\mu$ nor the canonical homotopy between $\mu$ and $g$ (see Remark 2.2) are good enough for us since we need a map definably homotopic to $f$ relative to $h$. We do as follows. Since $\left|K_{C}\right|$ and $|K| \backslash \operatorname{int}_{|K|}\left(\left|K_{E}\right|\right)$ are closed and disjoint, by Theorem 1.6 in [7], there exists a semialgebraic function $\lambda:|K| \rightarrow[0,1]$ such that $\lambda^{-1}(0)=\left|K_{C}\right|$ and $\lambda^{-1}(1)=|K| \backslash \operatorname{int}_{|K|}\left(\left|K_{E}\right|\right)$. Consider the map $H:|K| \times I \rightarrow|L|:(x, s) \mapsto$ $(1-s \lambda(x)) g(x)+s \lambda(x) \mu(x)$. The definable map $H$ is indeed continuous and is well-defined. Note that

$$
\left\{\begin{array}{l}
H(x, 0)=g(x) \quad \text { for all } \quad x \in|K| \\
H(x, s)=g(x)=h(x) \text { for all } \quad x \in\left|K_{C}\right| \text { and } s \in I .
\end{array}\right.
$$

Furthermore, observe that

$$
H(x, 1)=\left\{\begin{array}{l}
\mu(x) \text { for all } x \in|K| \backslash \operatorname{int}_{|K|}\left(\left|K_{E}\right|\right), \\
(1-\lambda(x)) \widetilde{h}(x)+\lambda(x) \mu(x) \text { for all } x \in\left|K_{E}\right|,
\end{array}\right.
$$


is semialgebraic. Hence $f \sim_{h} g \sim_{h} H(x, 1)$, as required.

As an immediate consequence of Theorem 3.1 we prove a more general result.

Corollary 3.3. Let $\left(X, A_{1}, \ldots, A_{k}\right)$ and $\left(Y, B_{1}, \ldots, B_{k}\right)$ be two systems of semialgebraic sets. Let $C$ be a relatively closed semialgebraic subset of $X$ and $h: C \rightarrow Y$ a semialgebraic map such that $h\left(C \cap A_{i}\right) \subset B_{i}, i=1, \ldots, k$. Then, if the subsets $A_{1}, \ldots, A_{k}$ are relatively closed in $X$, the map

$$
\begin{aligned}
\rho:\left[\left(X, A_{1}, \ldots, A_{k}\right),\left(Y, B_{1}, \ldots, B_{k}\right)\right]_{h}^{\mathcal{R}_{0}} & \rightarrow\left[\left(X, A_{1}, \ldots, A_{k}\right),\left(Y, B_{1}, \ldots, B_{k}\right)\right]_{h}^{\mathcal{R}} \\
{[f] } & \mapsto[f]
\end{aligned}
$$

is a bijection.

Proof. First note that the hypotheses of Lemma 2.1 do not include $X$ closed. Using that lemma (instead of its semialgebraic analogue) it can be shown (as in the proof of Theorem 3.1) that is enough to prove that $\rho_{1}:[X, Y]_{h}^{\mathcal{R}_{0}} \rightarrow$ $[X, Y]_{h}^{\mathcal{R}}:[f] \mapsto[f]$ is surjective. Let $(K, \phi)$ be a semialgebraic triangulation of $X$ partitioning $C$. Now, let $K_{C}=\{\sigma \in K: \phi(\sigma) \subset C\}$. We may assume that $K$ is the first barycentric subdivision of some simplicial complex. Since $\phi$ is a semialgebraic homeomorphism, it is enough to prove that the map $\rho_{2}:[|K|, Y]_{\widetilde{h}}^{\mathcal{R}_{0}} \rightarrow[|K|, Y]_{\widetilde{h}}^{\mathcal{R}}:[f] \mapsto[f]$ is surjective, where $\widetilde{h}=h \circ$ $\left.\phi\right|_{\left|K_{C}\right|}$. Observe that since $K_{C}$ is a relatively closed subcomplex of $K$ then $\operatorname{co}\left(K_{C}\right)=\operatorname{co}(K) \cap K_{C}$ (recall that the core $\operatorname{co}(K)$ of a simplicial complex $K$ is the unique maximal subcomplex of $K$ whose realization is closed in the ambient space). Since $K$ is the first barycentric subdivision of some simplicial complex, $\operatorname{co}(K)$ and $\operatorname{co}\left(K_{C}\right)$ are not empty. By Proposition III.1.6 in [8], there exists a semialgebraic strong deformation retract of $\left(|K|,\left|K_{C}\right|\right)$ to $\operatorname{co}\left(K, K_{C}\right):=\left(\operatorname{co}(K), \operatorname{co}\left(K_{C}\right)\right)$. Now the proof of Theorem 4.2 of Chapter III in [8], pp.253, applies in our context (using Lemma 2.1 instead of the semialgebraic homotopy extension lemma) and therefore, it is enough to prove that $\rho_{3}:[|\operatorname{co}(K)|, Y]_{\hat{h}}^{\mathcal{R}_{0}} \rightarrow[|\operatorname{co}(K)|, Y]_{\hat{h}}^{\mathcal{R}}:[f] \mapsto[f]$ is surjective, where $\hat{h}=\left.\widetilde{h}\right|_{\left|\operatorname{co}\left(K_{C}\right)\right|}$. Finally, since $|\operatorname{co}(K)|$ is closed and bounded, $\rho_{3}$ is surjective by Theorem 3.1.

Corollary 3.4. Let $X$ and $Y$ be two pairs of semialgebraic sets defined without parameters. Then there exist a bijection

$$
\rho:[X(\mathbb{R}), Y(\mathbb{R})] \rightarrow[X, Y]^{\mathcal{R}},
$$

where $[X(\mathbb{R}), Y(\mathbb{R})]$ denotes the classical homotopy set. Moreover, if $R$ contains the real field, then the result remains true for all semialgebraic sets $X$ and $Y$ defined with parameters in $\mathbb{R}$.

Proof. By Theorem III.5.1 in [8], there exits a canonical bijection between $[X(\mathbb{R}), Y(\mathbb{R})]$ and the semialgebraic homotopy set over the real algebraic 
numbers $[X(\overline{\mathbb{Q}}), Y(\overline{\mathbb{Q}})]^{\overline{\mathbb{Q}}}$. By Theorem III.4.2 in $[8]$, there exists a canonical bijection between $[X(\overline{\mathbb{Q}}), Y(\overline{\mathbb{Q}})]^{\overline{\mathbb{Q}}}$ and $[(X, A),(Y, B)]^{\mathcal{R}_{0}}$. The result then follows by Theorem 3.1. The proof of the second part is similar.

Remark 3.5. This corollary remains true for systems of semialgebraic sets satisfying the hypotheses of Corollary 3.3.

Corollary 3.6. Let $X$ and $Y$ be two definable sets defined without parameters. Then any definable map $f: X \rightarrow Y$ is definably homotopic to a definable map $g: X \rightarrow Y$ defined without parameters. If moreover $X$ and $Y$ are semialgebraic then $g$ can also be taken semialgebraic.

Proof. By the Triangulation Theorem there are triangulations of $X$ and $Y$ defined without parameters and therefore it suffices to prove the case in which both $X$ and $Y$ are semialgebraic. By Theorem 3.1, $f$ is definably homotopic to a semialgebraic map $g_{1}$. Finally, by Theorem III.3.1 in [8] applied to the real algebraic numbers $\overline{\mathbb{Q}}$ and $\mathcal{R}_{0}, g_{1}$ is semialgebraically homotopic to a semialgebraic map $g$ defined without parameters.

\section{The o-minimal homotopy groups}

We begin this section with a general discussion of homotopy groups in the o-minimal setting. Then we will relate the semialgebraic and the o-minimal homotopy groups via our Theorem 3.1. Finally, we will prove the usual properties related to homotopy in the o-minimal framework.

We will work with the category whose objects are the definable pointed sets, i.e., $\left(X, x_{0}\right)$, where $X$ is a definable set with $x_{0} \in X$, and whose morphisms are the definable continuous maps between definable pointed sets. In a similar way, we define the categories of definable pointed pairs, i.e., $\left(X, A, x_{0}\right)$, where $X$ is a definable set, $A$ is a definable subset of $X$ and $x_{0} \in A$.

Let $\left(X, x_{0}\right)$ be a definable pointed set. The o-minimal homotopy group of dimension $n, n \geq 1$, is the set $\pi_{n}\left(X, x_{0}\right)^{\mathcal{R}}=\left[\left(I^{n}, \partial I^{n}\right),\left(X, x_{0}\right)\right]^{\mathcal{R}}$. We define $\pi_{0}\left(X, x_{0}\right)$ as the set of definably connected components of $X$. The o-minimal relative homotopy group of dimension $n, n \geq 1$, of a definable pointed pair $\left(X, A, x_{0}\right)$ is the homotopy set $\pi_{n}\left(X, A, x_{0}\right)^{\mathcal{R}}=$ $\left[\left(I^{n}, I^{n-1}, J^{n-1}\right),\left(X, A, x_{0}\right)\right]^{\mathcal{R}}$, where $I^{n-1}=\left\{\left(t_{1}, \ldots, t_{n}\right) \in I^{n}: t_{n}=0\right\}$ and $J^{n-1}=\overline{\partial I^{n} \backslash I^{n-1}}$.

As in the classical case, we can define a group operation in the o-minimal homotopy groups $\pi_{n}\left(X, x_{0}\right)^{\mathcal{R}}$ and $\pi_{m}\left(X, A, x_{0}\right)^{\mathcal{R}}$ via the usual sum of maps for $n \geq 1$ and $m \geq 2$. Moreover, these groups are abelian for $n \geq 2$ and $m \geq 3$ (see pp. 340 and pp. 343 in [10]). Also, given a definable map between definable pointed sets (or pairs), we define the induced map in homotopy by the usual composing, which will be a homomorphism in the case we have a 
group structure. It is easy to check that with these definitions of o-minimal homotopy group and induced map, both the absolute and relative o-minimal homotopy groups $\pi_{n}(-)$ are covariant functors (see pp. 342 in [10]).

As a consequence of Theorem 3.1, we deduce the following relation between the semialgebraic and the o-minimal homotopy groups.

Theorem 4.1. For every semialgebraic pointed set $\left(X, x_{0}\right)$ and every $n \geq 1$, the map $\rho: \pi_{n}\left(X, x_{0}\right)^{\mathcal{R}_{0}} \rightarrow \pi_{n}\left(X, x_{0}\right)^{\mathcal{R}}:[f] \mapsto[f]$, is a natural isomorphism.

Proof. By Theorem $3.1 \rho$ is a bijection and its clearly a homomorphism. For the naturality condition, just observe that by definition the following diagram

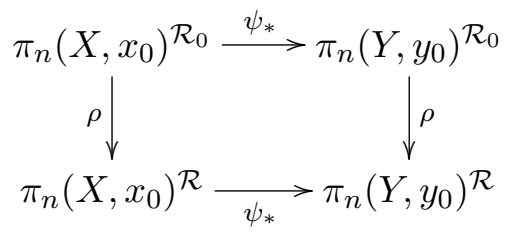

commutes, for every semialgebraic map $\psi:\left(X, x_{0}\right) \rightarrow\left(Y, y_{0}\right)$.

Remark 4.2. This last result remains true in the relative case and its proof is similar.

Corollary 4.3. The o-minimal homotopy groups are invariants under elementary extensions and o-minimal expansions.

Proof. The invariance under o-minimal expansions follows from the Triangulation Theorem and Theorem 4.1. The invariance under elementary extensions follows from Triangulation Theorem, Theorem 4.1 and the invariance of the semialgebraic homotopy sets under real closed field extensions (see Theorem III.6.3 in [8]).

The following result gives us a relation between the classical and the o-minimal homotopy groups (the case $n=1$ was already treated in [4]).

Corollary 4.4. Let $\left(X, x_{0}\right)$ be a semialgebraic pointed set defined without parameters. Then there exists a natural isomorphism between the classical homotopy group $\pi_{n}\left(X(\mathbb{R}), x_{0}\right)$ and the o-minimal homotopy group $\pi_{n}\left(X(R), x_{0}\right)^{\mathcal{R}}$ for every $n \geq 1$.

Proof. Either by Corollary 4.3 and Theorem III.6.4 in [8] or by Corollary 3.3 noting that the bijections involved are isomorphisms.

Remark 4.5. These last results remain true in the relative case and their proofs are similar. Moreover, the analogue of Corollary 4.3 is true for homotopy sets of definable systems satisfying the hypotheses of Corollary 3.3. 
Properties 4.6. The following properties of the o-minimal homotopy groups can be proved just adapting the proofs of their classical analogues and therefore we have not included them here. However, we give precise references of those proofs in the classical literature that can be adapted in an easy way (and, in particular, those that avoid the use of the path spaces with the compact-open topology).

1) The homotopy property: It is immediate that given two definably homotopic maps $\psi, \phi:\left(X, A, x_{0}\right) \rightarrow\left(Y, B, y_{0}\right)$, their induced homomorphisms $\psi_{*}, \phi_{*}: \pi_{n}\left(X, A, x_{0}\right)^{\mathcal{R}} \rightarrow \pi_{n}\left(Y, B, y_{0}\right)^{\mathcal{R}}$ are equal for every $n \geq 1$. Note that for $A=\left\{x_{0}\right\}$ and $B=\left\{y_{0}\right\}$ we have the absolute case.

2) The exactness property: Let $\left(X, A, x_{0}\right)$ be a pointed pair. For every $n \geq 2$ we define the boundary operator

$$
\begin{aligned}
\partial: \pi_{n}\left(X, A, x_{0}\right)^{\mathcal{R}} & \rightarrow \pi_{n-1}\left(A, x_{0}\right)^{\mathcal{R}} \\
{[f] } & \mapsto\left[\left.f\right|_{I^{n-1}}\right]
\end{aligned}
$$

For $n=1$, we define $\partial([u]),[u] \in \pi_{1}\left(X, A, x_{0}\right)^{\mathcal{R}}$, as the definably connected component of $A$ which contains $u(0)$. It is easy to prove that the boundary operator is a natural well-defined homomorphism for $n>1$. Moreover, if we denote by $i:\left(A, x_{0}\right) \rightarrow\left(X, x_{0}\right)$ and $j:\left(X, x_{0}, x_{0}\right) \rightarrow\left(X, A, x_{0}\right)$ the inclusion maps, then the following sequence is exact

$$
\cdots \rightarrow \pi_{n}\left(A, x_{0}\right) \stackrel{i_{*}}{\rightarrow} \pi_{n}\left(X, x_{0}\right) \stackrel{j_{*}}{\rightarrow} \pi_{n}\left(X, A, x_{0}\right) \stackrel{\partial}{\rightarrow} \pi_{n-1}\left(A, x_{0}\right) \rightarrow \cdots \rightarrow \pi_{0}\left(A, x_{0}\right),
$$

where the superscript $\mathcal{R}$ has been omitted. Indeed, by the triangulation theorem we can assume that $\left(X, A, x_{0}\right)$ is the realization of a simplicial complex with vertices in the real algebraic numbers. Then the exactness property follows from Corollary 4.4, the obvious fact that $\partial$ commutes with the isomorphism defined there and the classical exactness property.

3) The action of $\pi_{1}$ on $\pi_{n}$ : We can also define the usual action of $\pi_{1}(-)^{\mathcal{R}}$ on $\pi_{n}(-)^{\mathcal{R}}$. That is, given a pointed set $\left(X, x_{0}\right)$ and $[u] \in \pi_{1}\left(X, x_{0}\right)^{\mathcal{R}}$ there is a well-defined isomorphism $\beta_{[u]}: \pi_{n}\left(X, x_{0}\right)^{\mathcal{R}} \rightarrow \pi_{n}\left(X, x_{0}\right)^{\mathcal{R}}$ which only depends on $[u]$. In a similar way, given a pointed pair $\left(X, A, x_{0}\right)$ and $[u] \in \pi_{1}\left(A, x_{0}\right)^{\mathcal{R}}$ there is a well-defined isomorphism $\beta_{[u]}: \pi_{n}\left(X, A, x_{0}\right)^{\mathcal{R}} \rightarrow$ $\pi_{n}\left(X, A, x_{0}\right)^{\mathcal{R}}$ which only depends on $[u]$. The existence of both actions can be proved just adapting what is done in pp. 268 in [8] to the o-minimal setting. We briefly recall the construction of this action in the absolute case: given $[f] \in \pi_{n}\left(X, x_{0}\right)^{\mathcal{R}}$, we define $\beta_{[u]}([f]):=[H(t, 0)]$, where $H$ : $I^{n} \times I \rightarrow X$ is a definable homotopy such that $H(t, 1)=f(t)$ for all $t \in I^{n}$ and $H(t, s)=u(s)$ for all $t \in \partial I^{n}$ and $s \in[0,1]$ (note that Lemma 2.1 ensures the existence of this homotopy).

We will need the following technical lemma in the proof of the o-minimal Hurewicz theorem (in Section 5). We have included here its easy proof because of its cumbersome notation. 
Lemma 4.7. Let $\psi:\left(X, x_{0}\right) \rightarrow\left(Y, y_{0}\right)$ be a definable map between definable pointed sets and let $[u] \in \pi_{1}\left(X, x_{0}\right)^{\mathcal{R}}$. Then for all $[f] \in \pi_{n}\left(X, x_{0}\right)^{\mathcal{R}}$, $\psi_{*}\left(\beta_{[u]}([f])\right)=\beta_{\psi_{*}([u])}\left(\psi_{*}([f])\right)$.

Proof. It is enough to observe that if $H: I^{n} \times I \rightarrow X$ is a definable homotopy such that $H(t, 1)=f(t)$ for all $t \in I^{n}$ and $H(t, s)=u(s)$ for all $t \in \partial I^{n}$ and $s \in I$, then $\psi \circ H: I^{n} \times I \rightarrow Y$ is a definable homotopy such that $\psi \circ H(t, 1)=\psi \circ f(t)$ for all $t \in I^{n}$ and $\psi \circ H(t, s)=\psi \circ u(s)$ for all $t \in \partial I^{n}$ and $s \in I$.

4) The fibration property: We say that a definable map $p: E \rightarrow B$ is a definable (Serre) fibration if it has the definable homotopy lifting property for every (resp. closed and bounded) definable set $X$, i.e., if for each definable homotopy $H: X \times I \rightarrow B$ and each definable map $\widetilde{f}: X \rightarrow E$ with $p \circ \widetilde{f}(x)=H(x, 0)$ for all $x \in X$, there exists a definable homotopy $\widetilde{H}: X \times I \rightarrow E$ with $p \circ \widetilde{H}=H$ and $\widetilde{H}(x, 0)=\widetilde{f}(x)$ for all $x \in X$.

Remark 4.8. We say that a definable map $p: E \rightarrow B$ has the definable homotopy lifting property for a definable set $X$ relative to a definable subset $A$ of $X$ if for each definable homotopy $H: X \times I \rightarrow B$, each definable map $\widetilde{f}: X \rightarrow E$ with $p \circ \widetilde{f}(x)=H(x, 0)$ for all $x \in X$, and each definable homotopy $F: A \times I \rightarrow E$ with $p \circ F=\left.H\right|_{A \times I}$ there exists a definable homotopy $\widetilde{H}: X \times I \rightarrow E$ with $p \circ \widetilde{H}=H,\left.\widetilde{H}\right|_{A \times I}=F$ and $\widetilde{H}(x, 0)=\widetilde{f}(x)$ for all $x \in X$. As in the classical setting, the definable homotopy lifting property for a closed simplex $\sigma$ is equivalent to the definable homotopy lifting property for $\sigma$ relative to $\partial \sigma$. Indeed, there is a semialgebraic homeomorphism of $\sigma \times I$ onto itself which carries $\sigma \times\{0\}$ homeomorphically onto $(\sigma \times\{0\}) \cup(\partial \sigma \times I)$ (see the proof of Theorem III.3.1 in [11]). Therefore, the homotopy lifting property for closed simplices is equivalent to the homotopy lifting property for closed and bounded definable sets $X$ relative to closed subsets $A$ of $X$. For, by the triangulation theorem we can assume that $X$ is the realization of a closed simplicial complex and $A$ is the realization of a closed subcomplex of $X$. By induction over the skeleta of $X$ it suffices to construct a lifting over the closure of each open simplex contained in $X \backslash A$ at a time (and relative to the lifting constructed previously over its frontier).

With the above remark it is easy to adapt to the o-minimal setting the corresponding classical proof of the following fact (see Theorem 4.41 in [10]).

Theorem 4.9 (The fibration property). For every definable Serre fibration $p: E \rightarrow B$, the induced map $p_{*}: \pi_{n}\left(E, F, e_{0}\right) \rightarrow \pi_{n}\left(B, b_{0}\right)$ is a bijection for $n=1$ and an isomorphism for all $n \geq 2$, where $e_{0} \in F=p^{-1}\left(b_{0}\right)$.

As a consequence of the fibration property and the following proposition, we can extend Corollary 2.8 in [9], concerning coverings and the fundamental group, to all the homotopy groups (see Corollary 4.11 below). For a definition of definable covering see Section 2 in [9]. 
Proposition 4.10. Every definable covering $p: E \rightarrow B$ is a definable fibration.

Proof. Let $X$ be a definable set. Let $H: X \times I \rightarrow B$ a definable homotopy and $\widetilde{f}$ a definable map $\widetilde{f}: X \rightarrow E$ with $p \circ \widetilde{f}(x)=H(x, 0)$ for all $x \in X$. Consider the definable family of paths $\left\{H_{x}: x \in X\right\}$, where $H_{x}: I \rightarrow B: t \mapsto H(x, t)$. Since $p$ has the path lifting property (see Proposition 2.6 in [9]), for each $x \in X$ there is a (unique) lifting $\widetilde{H}_{x}: I \rightarrow E$ of $H_{x}$ such that $\widetilde{H}_{x}(0)=\widetilde{f}(x)$. Moreover, an easy modification of the proof of Proposition 2.6 in [9] shows that the family of paths $\left\{\widetilde{H}_{x}: x \in X\right\}$ is definable. Therefore, the map $\widetilde{H}: X \times I \rightarrow E:(x, t) \mapsto \widetilde{H}_{x}(t)$ is definable, $p \circ \widetilde{H}=H$ and $\widetilde{H}(x, 0)=\widetilde{f}(x)$ for all $x \in X$. It remains to prove that $\widetilde{H}$ is indeed continuous. Fix $\left(x_{0}, s_{0}\right) \in X \times I$. It is enough to prove that for each definable path $u: I \rightarrow X \times I$ with $u(1)=\left(x_{0}, s_{0}\right)$ we have that $\widetilde{H}(u(t)) \rightarrow \widetilde{H}\left(x_{0}, s_{0}\right)$ when $t \rightarrow 1$. We will prove it for $s_{0}=1$, but the same proof works for every $s_{0} \in I$.

Claim: We can assume that $u(0)=\left(x_{0}, 0\right)$, that $u$ is definably homotopic to the canonical path $I \rightarrow X \times I: t \mapsto\left(x_{0}, t\right)$ and that $\widetilde{H} \circ u:[0,1) \rightarrow E$ is continuous.

Granted the Claim, the path homotopy lifting property of $p$ (see Proposition 2.7 in [9]) implies that the respective liftings $\widetilde{H \circ u}$ and $\widetilde{H}_{x_{0}}$ of $H \circ u$ and $H_{x_{0}}$ starting at $\widetilde{f}\left(x_{0}\right)$, satisfy $\widetilde{H \circ u}(1)=\widetilde{H}_{x_{0}}(1)$. On the other hand, by the unicity of liftings of paths of $p$, we have that for every $\epsilon \in[0,1)$, $\widetilde{H}(u(t))=\widetilde{H \circ u}(t)$ for all $t \in[0, \epsilon]$. Therefore, $\widetilde{H}(u(t))=\widetilde{H \circ u}(t)$ for all $t \in[0,1)$. Hence, $\widetilde{H}(u(t)) \rightarrow \widehat{H \circ u}(1)=\widetilde{H}_{x_{0}}(1)=\widetilde{H}\left(x_{0}, 1\right)$ when $t \rightarrow 1$, as required.

Proof of the Claim: Since $X$ is definable, there exist a definably connected neighbourhood $U$ of $x_{0}$ which is definably contractible. Since $\widetilde{H} \circ u$ is definable, without loss of generality, $\widetilde{H} \circ u$ is continuous in $\left[\frac{2}{3}, 1\right)$ and $u(t) \in U \times I$ for all $t \in\left[\frac{2}{3}, 1\right)$. Let $\left(x_{1}, s_{1}\right)=u\left(\frac{2}{3}\right)$. Take a definable path $w:\left[0, \frac{1}{3}\right] \rightarrow U$ such that $w(0)=x_{0}$ and $w\left(\frac{1}{3}\right)=x_{1}$. We define the path $\hat{u}(t): I \rightarrow X \times I$ such that $\hat{u}(t):=(w(t), 0)$ for all $t \in\left[0, \frac{1}{3}\right], \hat{u}(t):=\left(x_{1}, 3 s_{1}\left(t-\frac{1}{3}\right)\right)$ for all $t \in\left[\frac{1}{3}, \frac{2}{3}\right]$ and $\hat{u}(t):=u(t)$ for all $t \in\left[\frac{2}{3}, 1\right]$. The definable path $\widetilde{H}(\hat{u}(t))$ is continuous for all $t \in[0,1)$ because $\widetilde{f}$ is continuous and because of the construction of $\widetilde{H}$. Since $U$ is definably contractible, $\left\{x_{0}\right\} \times I$ is a definable deformation retract of $U \times I$ and therefore $\hat{u}$ is definably homotopic to the canonical path $I \rightarrow X \times I: t \mapsto\left(x_{0}, t\right)$. Finally, since we are just interested in the behaviour of the definable path $u$ when $t$ is near 1 , we can replace $u$ by $\hat{u}$.

Corollary 4.11. Let $p: E \rightarrow B$ be a definable covering and let $p\left(e_{0}\right)=b_{0}$. Then $p_{*}: \pi_{n}\left(E, e_{0}\right)^{\mathcal{R}} \rightarrow \pi_{n}\left(B, b_{0}\right)^{\mathcal{R}}$ is an isomorphism for every $n>1$ and injective for $n=1$. 
Proof. Since $p^{-1}\left(b_{0}\right)$ is finite, we have that $\pi_{n}\left(p^{-1}\left(b_{0}\right), e_{0}\right)=0$ for every $n \geq 1$. Then the result follows from Proposition 4.10 and both the exactness and the fibration properties.

\section{The o-minimal Hurewicz theorems and the o- minimal Whitehead theorem}

Next we will prove both the absolute and relative Hurewicz theorems in the o-minimal setting by transferring from the semialgrebraic setting via Theorem 3.1.

First let us define the o-minimal Hurewicz homomorphism. Recall that there exists an o-minimal singular homology theory $H_{*}(-)^{\mathcal{R}}$ on the category of definable sets (see [13]). Moreover, by Proposition 3.2 in [4] there exists a natural isomorphism $\theta$ between the functors $H_{*}(-)^{\mathcal{R}_{0}}$ and $H_{*}(-)^{\mathcal{R}}$ on the category of (pairs of) semialgebraic sets (note that the notation used in the above paper is different from ours, where $H_{*}(-)^{\mathcal{R}_{0}}=H_{*}^{s a}(-)$ and $\left.H_{*}(-)^{\mathcal{R}}=H_{*}^{\text {def }}(-)\right)$. Fix $n \geq 1$. By Proposition 3.2 in [4],

$$
H_{n}\left(I^{n}, \partial I^{n}\right)^{\mathcal{R}_{0}} \cong H_{n}\left(I^{n}(\mathbb{R}), \partial I^{n}(\mathbb{R})\right) \cong \mathbb{Z} .
$$

We fix a generator $z_{n}^{\mathcal{R}_{0}}$ of $H_{n}\left(I^{n}, \partial I^{n}\right)^{\mathcal{R}_{0}}$ and we define $z_{n}^{\mathcal{R}}:=\theta\left(z_{n}^{\mathcal{R}_{0}}\right)$. Now, given a definable pointed set $\left(X, x_{0}\right)$, the o-minimal Hurewicz homomorphism, for $n \geq 1$, is the map $h_{n, \mathcal{R}}: \pi_{n}\left(X, x_{0}\right)^{\mathcal{R}} \rightarrow H_{n}(X)^{\mathcal{R}}:[f] \mapsto$ $h_{n, \mathcal{R}}([f])=f_{*}\left(z_{n}^{\mathcal{R}}\right)$, where $f_{*}: H_{n}\left(I^{n}, \partial I\right)^{\mathcal{R}} \rightarrow H_{n}(X)^{\mathcal{R}}$ denotes the map in o-minimal singular homology induced by $f$. Note that by the homotopy axiom of o-minimal singular homology if $f \sim g$ then $f_{*}=g_{*}$, hence $h_{n, \mathcal{R}}$ is well-defined. We define the relative Hurewicz homomorphism adapting in the obvious way what was done in the absolute case. Now, following the classical proof, it is easy to check that $h_{n, \mathcal{R}}$ is a natural transformation between the functors $\pi_{n}(-)^{\mathcal{R}}$ and $H_{n}(-)^{\mathcal{R}}$ (see Proposition V.4.1 in [11]). This fact can also be deduced from the semialgebraic setting (see Remark $5.2)$.

The following result give us a relation between the semialgebraic and the o-minimal Hurewicz homomorphisms.

Proposition 5.1. Let $\left(X, x_{0}\right)$ be a semialgebraic pointed set. Then the following diagram commutes

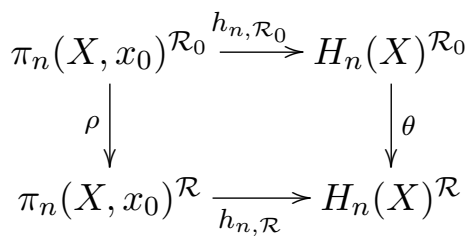

for all $n \geq 1$. 
Proof. Let $[f] \in \pi_{n}\left(X, x_{0}\right)^{\mathcal{R}_{0}}$. By definition $z_{n}^{\mathcal{R}}=\theta\left(z_{n}^{\mathcal{R}_{0}}\right)$ and by the naturality of $\theta$ we have that $\theta\left(f_{*}\left(z_{n}^{\mathcal{R}_{0}}\right)\right)=f_{*}\left(\theta\left(z_{n}^{\mathcal{R}_{0}}\right)\right)$. Therefore $\theta\left(h_{n, \mathcal{R}_{0}}([f])\right)=$ $\theta\left(f_{*}\left(z_{n}^{\mathcal{R}_{0}}\right)\right)=f_{*}\left(\theta\left(z_{n}^{\mathcal{R}_{0}}\right)\right)=f_{*}\left(z_{n}^{\mathcal{R}}\right)=h_{n, \mathcal{R}}(\rho([f]))$.

Remark 5.2. (1) This last result remains true in the relative case and its proof is similar.

(2) Since $h_{n, \mathcal{R}_{0}}$ is a homomorphism for $n \geq 1$ (see Theorem III.7.3 in [8]), it follows from Proposition 5.1 and the Triangulation theorem that $h_{n, \mathcal{R}}$ is also a homomorphism for $n \geq 1$.

Theorem 5.3 (The o-minimal Hurewicz theorems). Let $\left(X, x_{0}\right)$ be a definable pointed set and $n \geq 1$. Suppose that $\pi_{r}\left(X, x_{0}\right)^{\mathcal{R}}=0$ for every $0 \leq r \leq n-1$. Then the o-minimal Hurewicz homomorphism

$$
h_{n, \mathcal{R}}: \pi_{n}\left(X, x_{0}\right)^{\mathcal{R}} \rightarrow H_{n}(X)^{\mathcal{R}}
$$

is surjective and it induces an isomorphism between the abelianization of $\pi_{n}\left(X, x_{0}\right)^{\mathcal{R}}$ and $H_{n}(X)^{\mathcal{R}}$. In particular, $h_{n, \mathcal{R}}$ is an isomorphism for $n \geq 2$.

Proof. It is enough to show that $h_{n, \mathcal{R}}$ is surjective and its kernel is the subgroup generated by $\left\{\beta_{[u]}([f])[f]^{-1}:[u] \in \pi_{1}\left(X, x_{0}\right)^{\mathcal{R}},[f] \in \pi_{n}\left(X, x_{0}\right)^{\mathcal{R}}\right\}$, where $\beta_{[u]}$ is the action of $\pi_{1}$ on $\pi_{n}$ defined in Properties 4.6.3. Indeed, if $n \geq 2$ then by hypothesis $\pi_{1}\left(X, x_{0}\right)^{\mathcal{R}}=0$ and therefore the kernel of $h_{n, \mathcal{R}}$ would be trivial. If $n=1$ then $\operatorname{Ker}\left(h_{1, \mathcal{R}}\right)$ would be the subgroup generated by $\left\{\beta_{[u]}([v])[v]^{-1}:[u] \in \pi_{1}\left(X, x_{0}\right)^{\mathcal{R}},[v] \in \pi_{1}\left(X, x_{0}\right)^{\mathcal{R}}\right\}$. On the other hand, using the definable homotopy $H(t, s)=u(t s) v(t) u(s-t s)$, we have that $\beta_{[u]}([v])[v]^{-1}=[u][v][u]^{-1}[v]^{-1}$. Hence, $\operatorname{Ker}\left(h_{1, \mathcal{R}}\right)$ would be the commutator of $\pi_{1}\left(X, x_{0}\right)^{\mathcal{R}}$.

Note that $X$ is definably connected since $\pi_{0}\left(X, x_{0}\right)^{\mathcal{R}}=0$. Let $(K, \phi)$ be a definable triangulation of $X$ and $y_{0}=\phi^{-1}\left(x_{0}\right)$. Since $\pi_{r}(-)^{\mathcal{R}}$ is a covariant functor, $\pi_{r}\left(|K|, y_{0}\right)^{\mathcal{R}}=0$ for $0 \leq r \leq n-1$. Moreover, as $\rho$ is a natural isomorphism, $\pi_{r}\left(|K|, y_{0}\right)^{\mathcal{R}_{0}} \cong \pi_{r}\left(|K|, y_{0}\right)^{\mathcal{R}}=0$ for $0 \leq r \leq n-1$. Since $h_{n, \mathcal{R}}$ is a natural transformation, the following diagram

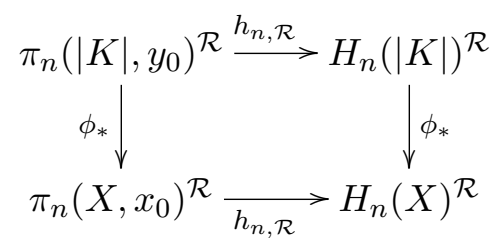

commutes. Furthermore, since $\phi$ is a homeomorphism, the induced map $\phi_{*}$ in both homology and homotopy are isomorphisms. Hence, by Lemma 4.7 , it is enough to prove that $h_{n, \mathcal{R}}: \pi_{n}\left(|K|, y_{0}\right)^{\mathcal{R}} \rightarrow H_{n}(|K|)^{\mathcal{R}}$ is surjective and that its kernel is the subgroup generated by $\left\{\beta_{[u]}([f])[f]^{-1}:[u] \in\right.$ 
$\left.\pi_{1}\left(|K|, y_{0}\right)^{\mathcal{R}},[f] \in \pi_{n}\left(|K|, y_{0}\right)^{\mathcal{R}}\right\}$. By Proposition 5.1, the following diagram

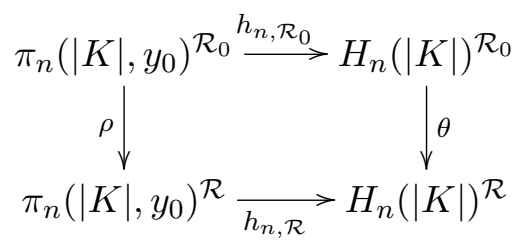

commutes. Since $\rho$ and $\theta$ are natural isomorphism, it is enough to prove that $h_{n, \mathcal{R}_{0}}: \pi_{n}\left(|K|, y_{0}\right)^{\mathcal{R}_{0}} \rightarrow H_{n}(|K|)^{\mathcal{R}_{0}}$ is surjective and that its kernel is the subgroup generated by $\left\{\beta_{[u]}([f])[f]^{-1}:[u] \in \pi_{1}\left(|K|, y_{0}\right)^{\mathcal{R}_{0}},[f] \in\right.$ $\left.\pi_{n}\left(|K|, y_{0}\right)\right\}^{\mathcal{R}_{0}}$. But this fact follows from the semialgebraic Hurewicz theorems (see Theorem III.7.4 in [8]).

Theorem 5.4 (The o-minimal relative Hurewicz theorems). Let $\left(X, A, x_{0}\right)$ be a definable pointed pair and $n \geq 2$. Suppose that $\pi_{r}\left(X, A, x_{0}\right)^{\mathcal{R}}=0$ for every $1 \leq r \leq n-1$. Then the o-minimal Hurewicz homomorphism $h_{n, \mathcal{R}}$ : $\pi_{n}\left(X, A, x_{0}\right)^{\mathcal{R}} \rightarrow H_{n}(X, A)^{\mathcal{R}}$ is surjective and its kernel is the subgroup generated by $\left\{\beta_{[u]}([f])[f]^{-1}:[u] \in \pi_{1}\left(A, x_{0}\right)^{\mathcal{R}},[f] \in \pi_{n}\left(X, A, x_{0}\right)^{\mathcal{R}}\right\}$. In particular, $h_{n, \mathcal{R}}$ is an isomorphism for $n \geq 3$.

Proof. It is enough to adapt the proof of the o-minimal absolute Hurewicz theorems to the relative case. Note that at some point, we need the relative version of Lemma 4.7 (whose proof is similar), i.e., that given a definable map $\psi:\left(X, A, x_{0}\right) \rightarrow\left(Y, B, y_{0}\right)$ and $[u] \in \pi_{1}\left(A, x_{0}\right)^{\mathcal{R}}$, we have that $\psi_{*}\left(\beta_{[u]}([f])\right)=\beta_{\psi_{*}([u])}\left(\psi_{*}([f])\right)$ for all $[f] \in \pi_{n}\left(X, A, x_{0}\right)^{\mathcal{R}}$.

Remark 5.5. (1) In Theorem 5.1 in [9] it is proved that the abelianization of $\pi_{n}\left(X, x_{0}\right)^{\mathcal{R}}$ and $H_{n}(X)^{\mathcal{R}}$ are isomorphic for $\left(X, x_{0}\right)$ a definably connected definable pointed set. In the case $n=1$ in Theorem 5.3 we give an explicit natural isomorphism.

(2) With the hypotheses of Theorem 5.4, $\pi_{2}\left(X, A, x_{0}\right)^{\mathcal{R}} / \operatorname{Ker}\left(h_{2, \mathcal{R}}\right)$ $\cong H_{2}(X, A)^{\mathcal{R}}$ is abelian and therefore $\operatorname{Ker}\left(h_{2, \mathcal{R}}\right)$ contains the commutator subgroup of $\pi_{2}\left(X, A, x_{0}\right)^{\mathcal{R}}$. This fact can also be shown directly by proving that for every $[f],[g] \in \pi_{2}\left(X, A, x_{0}\right)^{\mathcal{R}},[g][f][g]^{-1}=\beta_{[u]}([f])$, where $u(t)=g(t, 0)$ for $t \in I$.

We finish this section with the proof of the o-minimal Whitehead theorem. We say that a definable map $f: X \rightarrow Y$ is a definable homotopy equivalence if there exist a definable map $f^{\prime}: Y \rightarrow X$ such that $f \circ f^{\prime} \sim \operatorname{id}_{Y}$ and $f^{\prime} \circ f \sim \operatorname{id}_{X}$. Note that if a definable map $f$ is a definable homotopy equivalence then it is a definable homotopy equivalence relative to a point. Indeed, it suffices to adapt the classical proof using Lemma 2.1 instead of the lifting property (see Proposition 0.19 in [10]). 
Theorem 5.6 (The o-minimal Whitehead theorem). Let $X$ and $Y$ be two definably connected sets. Let $f: X \rightarrow Y$ be a definable map such that for some $x_{0} \in X, f_{*}: \pi_{n}\left(X, x_{0}\right)^{\mathcal{R}} \rightarrow \pi_{n}\left(Y, f\left(x_{0}\right)\right)^{\mathcal{R}}$ is an isomorphism for all $n \geq 1$. Then $f$ is a definable homotopy equivalence.

Proof. Let $\left(K, \phi_{1}\right)$ and $\left(L, \phi_{2}\right)$ be definable triangulations of $X$ and $Y$, respectively. Consider the points $x_{1}=\phi_{1}^{-1}\left(x_{0}\right)$ and $y_{1}=\phi_{2}^{-1}\left(f\left(x_{0}\right)\right)$. It suffices to prove that the definable map $\widetilde{f}=\phi_{2}^{-1} \circ f \circ \phi_{1}:|K| \rightarrow|L|$ is a definable homotopy equivalence provided $\tilde{f}_{*}: \pi_{n}\left(|K|, x_{1}\right)^{\mathcal{R}} \rightarrow \pi_{n}\left(|L|, y_{1}\right)^{\mathcal{R}}$ is an isomorphism for all $n \geq 1$. By Theorem 3.1 there exists a semialgebraic map $g:\left(|K|, x_{1}\right) \rightarrow\left(|L|, y_{1}\right)$ such that $g \sim \widetilde{f}$. By the homotopy property it follows that $g_{*}=\widetilde{f}_{*}: \pi_{n}\left(|K|, x_{1}\right)^{\mathcal{R}} \rightarrow \pi_{n}\left(|L|, y_{1}\right)^{\mathcal{R}}$ is an isomorphism for all $n \geq 1$. Therefore by Theorem 4.1, $g_{*}: \pi_{n}\left(|K|, x_{1}\right)^{\mathcal{R}_{0}} \rightarrow \pi_{n}\left(|L|, y_{1}\right)^{\mathcal{R}_{0}}$ is an isomorphism for all $n \geq 1$. Hence, by Theorem III.6.6 in [8], $g$ is a semialgebraic homotopy equivalence, that is, there exists a semialgebraic $\operatorname{map} g^{\prime}:|L| \rightarrow|K|$ such that $\operatorname{id}_{|K|} \sim_{0} g^{\prime} \circ g$ and $\operatorname{id}_{|L|} \sim_{0} g \circ g^{\prime}$, where $\sim_{0}$ means "semialgebraically homotopic". Hence $\operatorname{id}_{|K|} \sim_{0} g^{\prime} \circ g \sim g^{\prime} \circ \widetilde{f}$ and so $\operatorname{id}_{|K|} \sim g^{\prime} \circ \tilde{f}$. In a similar way we prove that $\operatorname{id}_{|L|} \sim \widetilde{f} \circ g^{\prime}$. Therefore $\tilde{f}$ is a definable homotopy equivalence, as required.

Corollary 5.7. Let $X$ be a definable set and let $x_{0} \in X$. If $\pi_{n}\left(X, x_{0}\right)^{\mathcal{R}}=0$ for all $n \geq 0$ then $X$ is definably contractible.

Proof. This follows from Theorem 5.6 applied to a constant map.

Next result follows the transfer approach developed in [5].

Corollary 5.8. Let $X$ be a semialgebraic set defined without parameters. Then $X$ is definably contractible if and only if $X(\mathbb{R})$ is contractible in the classical sense.

Proof. This follows from Corollary 4.4 and Corollary 5.7.

\section{References}

[1] E. Baro, Normal triangulations in o-minimal structures, preprint, 15pp., 2007, www.uam.es/elias.baro/articulos.html.

[2] E. Baro and M. Otero, Locally definable homotopy, preprint, 33pp., 2008, www. uam.es/elias.baro/articulos.html.

[3] A. Berarducci, M. Mamino and M. Otero, Higher homotopy of groups definable in o-minimal structures, 2008 Preprint, arXiv:0809.4940 [math.LO].

[4] A. Berarducci and M. Otero, o-minimal fundamental group, homology and manifolds, J. London Math. Soc. (2) 65 (2002), no. 2, 257-270. 
[5] A. Berarducci and M. Otero, Transfer methods for o-minimal topology, J. Symb. Log. 68 (2003) 785-794.

[6] L. van den Dries, Tame topology and o-minimal structures, London Mathematical Society Lecture Note Series, 248, Cambridge University Press, 1998.

[7] H. Delfs and M.Knebusch, Separation, retractions and homotopy extension in semialgebraic spaces, Pacific J. Math. 114 (1984), no. 1, $47-71$.

[8] H. Delfs and M. Knebusch, Locally semialgebraic spaces, Lecture Notes in Mathematics, 1173, Springer-Verlag, Berlin, 1985.

[9] M. Edmundo and M. Otero, Definably compact abelian groups, J. Math. Log. 4 (2004), no. 2, 163-180.

[10] A. Hatcher, Algebraic topology, Cambridge University Press, 2002.

[11] S. Hu, Homotopy theory, Pure and Applied Mathematics, Vol. VIII Academic Press, New York-London 1959.

[12] A.Piȩkosz, O-minimal homotopy and generalized (co)homology, preprint, 2008.

[13] A. Woerheide, O-minimal homology, PhD Thesis, University of Illinois at Urbana-Champaign, 1996.

DEPARTAMENTO DE MATEMÁTICAS, UNIVERSIDAD AUTÓNOMA DE MADRID, 28049, MADRID, SPAIN.

E-mail address: elias.baro@uam.es

DEPARTAMENTO DE MATEMÁTICAS, UNIVERSIDAD AUTÓNOMA DE MADRID, 28049, MADRID, SPAIN.

E-mail address: margarita.otero@uam.es 